\title{
A00-39805
}

AIAA Paper 2000-4420

\section{SOLAR SAIL ORBIT OPERATIONS AT ASTEROIDS*}

\author{
Esther Morrow ${ }^{\dagger}$ \\ D. J. Scheeres $\ddagger$ \\ Dan Lubin $\S$ \\ June 19,2000
}

\begin{abstract}
The inherent capabilities of solar sails and the fact that they need no onboard supplies of fuel for propulsion make them well suited for use in long-term, multiple-objective missions. They are especially well suited for the exploration of asteroids, where one spacecraft could rendezvous with a number of asteroids in succession. The orbital mechanics of solar sail operations about an asteroid, however, have not yet been studied in detail. Building on previous studies that consider the equations of motion, we find both hovering points and orbiting trajectories about various sized asteroids using equations of motion for a solar sail spacecraft. These hovering points are stabilizable using feedback control to sail attitude alone. The orbiting trajectories are stable and offer good coverage of the asteroid surface, although restrictions on sail acceleration are needed for smaller asteroids.
\end{abstract}

\section{Introduction}

The concept of the solar sail has been known for more than a century, but it has only been with the advent of micro-technologies and thin films that solar sailing appears to be a practical mode of spaceflight ${ }^{3}$. Because of the long history and proven usefulness of conventional spacecraft, solar sails will never be considered as a replacement technology for conventional propulsion; however, there are some mission applications for which sails are particularly well-suited. Long-term, multiple-objective missions

\footnotetext{
- Copyright (C)2000 The American Institute of Aeronautics and Astronautics Inc. All rights reserved.

${ }^{\dagger}$ Research Associate, California Space Institute, Scripps Institute of Oceanography, University of California, San Diego, La Jolla, CA

IAssistant Professor, Department of Aerospace Engineering, 'The University of Michigan, Ann Arbor, MI

$\S$ Associate Research Physicist, California Space Institute, Scripps Institute of Oceanography, University of California, San Diego, La Jolla, CA
}

such as those to more than one asteroid is just such an application. These missions have not yet been studied in detail and, in particular, solar sail behavior about asteroids has not been studied. This paper is a first step in this direction.

Relying on solar propulsion instead of convential propulsion allows us flexibility in studying asteroids. With a solar sail spacecraft, we are not limited by carrying onboard supplies of fuel for propulsion. Depending on sail performance in the space environment, several asteroids could be visited in succession and orbited for extended periods of time. Thus, a spacecraft of this type could enhance our understanding of asteroids by shortening the period of time between missions.

We derive a model for solar sail dynamics about an asteroid and consider a number of possible options for operations in the asteroid environment. We find limits on feasible sail operations as a function of sail parameters, asteroid parameters, and asteroid orbit. Both orbital and hovering options are considered. For the purposes of this study, we focus only on the behavior of the solar sail spacecraft after rendezvous has been achieved.

For our model we assume a spherical, point mass asteroid, a perfectly reflecting solar sail, and a circular asteroid orbit about the sun. These assumptions can be relaxed in future studies. We first develop the basic equations of motion for the system. We then consider the constraints and feasibility of hovering sail trajectories, complementing the studies made by McInnes ${ }^{2}$. We find a continuum of hovering points which would allow station-keeping for long periods of time. At these points, the spacecraft could hover while a lander deploys to the surface to collect samples or where the craft could monitor the asteroid at a particular phase angle for extended lengths of time. Next, we consider the constraints and feasibility of orbital operations about an asteroid, from which we develop a number of basic criteria for necessary sail acceleration as a function of aster- 
oid parameters. The orbital trajectories are found to be stable and to offer good coverage of the asteroid surface. These trajectories would be very useful for mapping purposes.

\section{Model}

To model the dynamics of a sail close to an asteroid we can apply the Hill approximation with appropriate generalizations to account for the solar sail's force vector. The Hill approximation applies very well to the motion of spacecraft about asteroids, due to the small mass of asteroids relative to the sun, and due to the proximity that the craft maintains to the asteroid $^{6}$. The Hill approximation can also be easily modified to account for the effect of solar radiation pressure $^{8}$.

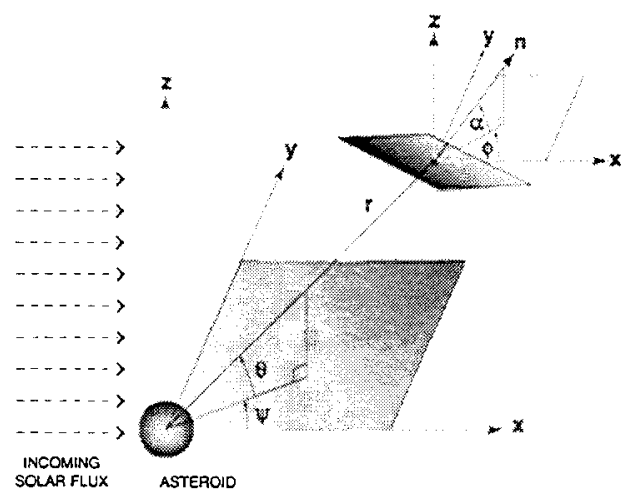

Figure 1: Model schematic for a solar sail spacecraft about an asteroid.

With the Hill approximation the equations of motion for a spacecraft including the solar radiation pressure are:

$$
\begin{aligned}
\ddot{x}-2 \Omega \dot{y} & =-\frac{\mu}{r^{3}} x+3 \Omega^{2} x+a_{x} \\
\ddot{y}+2 \Omega \dot{x} & =-\frac{\mu}{r^{3}} y+a_{y} \\
\ddot{z} & =-\frac{\mu}{r^{3}} z-\Omega^{2} z+a_{z}
\end{aligned}
$$

where the origin of the rotating reference frame is centered at the asteroid with the positive $x$-axis in the anti-solar direction, the $z$-axis normal to the asteroid orbit ecliptic, and the $y$-axis according to the right hand rule. This frame rotates about the $z$-axis with angular velocity $\Omega=\sqrt{\mu_{\text {sun }} / a_{a s t}^{3}}$, where $\mu_{\text {sun }}$ is the sun's gravitational parameter $\left(\sim 1.34 \times 10^{11}\right.$ $\left.\mathrm{km}^{3} / \mathrm{s}^{2}\right)$, and $a_{a s t}$ is the asteroid's heliocentric orbit radius in $\mathrm{km}$. The gravitational parameter of the asteroid is denoted as $\mu$.
The spacecraft is propelled by solar radiation flux incident on the sail, which is herein assumed to be perfectly reflecting and planar. The sail receives an action force from incident sunlight and a reaction force from the reflected light so that the net force is directed normal to the surface of the sail and pointing away from the sun. The acceleration vector of this force is denoted as $\mathbf{a}=\left[\begin{array}{lll}a_{x x} & a_{y} & a_{z}\end{array}\right]=a_{p}(\mathbf{l} \cdot \mathbf{n})^{2} \mathbf{n}$.

The unit vector 1 defines the direction of the incident light, while $\mathbf{n}$ is the unit normal to the sail. In the rotating reference frame 1 will always equal $\left[\begin{array}{lll}1 & 0 & 0\end{array}\right]$ and $\mathbf{n}$ will be $[\cos \phi \cos \alpha \sin \phi \cos \alpha \sin \alpha]$, where $\alpha$ is the sail pitch angle with respect to the sun-line and $\phi$ is the orientation angle about the $z$ axis. The direction of the SRP force can never be pointed toward the sun so the sail attitude is constrained such that $\mathbf{l} \cdot \mathbf{n} \geq 0$.

The parameter $a_{p}$ is called the characteristic acceleration and is represented as

$a_{p}=\frac{2 P}{c R_{o}^{2}} \frac{R_{o}^{2}}{R^{2}} \frac{1}{\sigma}$

for a perfectly reflecting solar sail. $P / R_{o}^{2}$ is the solar radiation intensity $\left(1368 \mathrm{~W} / \mathrm{m}^{2}\right.$ at $\left.1 \mathrm{AU}\right), c$ is the speed of light $\left(3 \times 10^{8} \mathrm{~m} / \mathrm{s}\right), R_{o}$ is the distance from the sun to the earth $\left(1.5 \times 10^{8} \mathrm{~km}\right), R$ is the distance from the sun to the sail in $\mathrm{km}$, and $\sigma$ is the mass to area ratio of the sail $\left(\mathrm{kg} / \mathrm{m}^{2}\right)$ or sail loading. At 1 $\mathrm{AU}$

$a_{p}=a_{p_{\circ}}=\frac{2 P}{c R_{o}^{2}} \frac{1}{\sigma}$

and, in general,

$a_{p}=a_{p o} \frac{R_{o}^{2}}{R^{2}}$

Thus, SRP varies as $1 / R^{2}$ about the sun (but see discussion in McInnes ${ }^{3}$ ).

Multiplying and dividing by the sun's gravitational parameter $\mu_{\text {sur }}$, we have

$a_{p}=\frac{2 P}{c \mu_{\text {sun }}} \frac{\mu_{\text {sun }}}{R^{2}} \frac{1}{\sigma}$

$a_{p}=\beta \frac{\mu_{\text {sun }}}{R^{2}}$

where $\beta=\sigma^{*} / \sigma$ is the nondimensional sail loading parameter, called the sail lightness number, defined to be the ratio of the solar radiation pressure acceleration to the solar gravitational acceleration. The critical sail loading parameter $\sigma^{*}=2 P / c \mu_{s u n}$ is a constant whose value is approximately $1.53 \mathrm{~g} / \mathrm{m}^{2}$.

The value of the characteristic acceleration varies with both the efficiency and size of the sail ${ }^{3}$. For 
example, McInnes ${ }^{3}$ shows that a typical value for a $98 \times 98 \mathrm{~m}$ square sail which carries a payload of $25 \mathrm{~kg}$ is $1 \mathrm{~mm} / \mathrm{s}^{2}$. At this acceleration, a solar sail spacecraft has trip times to asteroids comparable to conventional spacecraft. It yields a sail loading parameter of $9.12 \mathrm{~g} / \mathrm{m}^{2}$, or a sail lightness number of 0.1678 . This parameter can be reached with a sail film thickness of $2 \mu \mathrm{m}$.

\section{Solar sail hovering constraints and dynamics}

Given the basic equations of motion, we search for conditions under which the spacecraft can be placed into an artificial equilibrium point - found by setting all the time derivatives to zero and finding the combination of SRP acceleration and natural forces that will yield no net acceleration acting on the sail. This is a specialization of the analysis carried out by McInnes ${ }^{2}$ for the restricted 3-body problem, now applied to the Hill problem. Following the Hill equations we find the equations of motion of a solar sail spacecraft to be as in (1)-(3).

Because the sail normal is required to point in the anti-solar direction, we are limited to $-\pi / 2 \leq$ $\alpha \leq \pi / 2$. The problem is symmetric about the angle $\alpha=0$, however, so we need only consider $0 \leq \alpha \leq \pi / 2$. When $\alpha=\pi / 2$ the sail is edge on to the sun. In this situation, we effectively are "in irons," i.e., have no SRP component at all. While there may be instances when this effect would be desirable, we do not consider this case explicitly. Thus, we limit our discussion to $0 \leq \alpha<\pi / 2$.

Likewise, in order to satisfy the constraint $\mathbf{l} \cdot \mathbf{n} \geq 0$, we only consider $\phi$ limited to $-\pi / 2 \leq \phi \leq \pi / 2$. The problem is also symmetric about $\phi=0$; therefore, we consider $\phi$ such that $0 \leq \phi<\pi / 2$ for the same reasons stated for $\alpha$ above.

The preceding definitions lead us to, in the most general case, define the the equations of motion for hovering points to be:

$0=-\frac{\mu}{r^{3}} x+3 \Omega^{2} x+a_{x}$

$0=-\frac{\mu}{r^{3}} y+a_{y}$

$0=-\frac{\mu}{r^{3}} z-\Omega^{2} z+a_{z}$

with the components of a as

$a_{x}=a_{p} \cos ^{2} \phi \cos ^{2} \alpha \cos \phi \cos \alpha$

$a_{y}=a_{p} \cos ^{2} \phi \cos ^{2} \alpha \sin \phi \cos \alpha$

$a_{z}=a_{p} \cos ^{2} \phi \cos ^{2} \alpha \sin \alpha$

and the vector $\mathbf{r}$ as

$\mathbf{r}=r[\cos \theta \cos \psi \cos \theta \sin \psi \sin \theta]$ where $\theta$ is the declination angle measured from the $x y$-plane toward the $z$-axis and $\psi$ is the right ascension angle measured in the $x y$-plane from the $+x$ axis.

\section{Hovering points with no SRP force}

We first consider the hovering points for the system with no SRP force. In this case, $a_{x}=a_{y}=a_{z}=0$. It is obvious that $y$ and $z$ must also be 0 to satisfy (10) and (11), respectively. Thus, from (9), $\mu / r^{3}=$ $3 \Omega^{2}$ at equilibrium. Solving for $r$, we find

$r= \pm\left(\frac{\mu}{3 \Omega^{2}}\right)^{1 / 3}$

The value $\left(\frac{\mu}{3 \Omega^{2}}\right)^{1 / 3}$ is called the Hill radius and denoted $r_{H}$ herein. We use this radius to normalize the hovering point radii throughout our analysis.

\section{Hovering points along the $x$-axis}

We consider next the simplest situation for the model, when the sail is along the $x$-axis. Here, $\phi$, $\psi$ and $\alpha$ are all 0 since there are no components in the $y$ or $z$ directions. In this case, the equations of motion become:

$0=-\frac{\mu}{r^{3}} x+3 \Omega^{2} x+a_{p}$

which becomes

$\frac{x}{|x|^{3}}=\frac{a_{p}}{\mu}\left(\frac{1}{1-r_{n}^{3}}\right)$

where $r_{n}$ is the nondimensionalized value for $r$ normalized by the Hill radius $r_{H}$, i.e., $r_{n}=r / r_{H}$.

In this case there are two equilibrium points, one for $x$ positive and one for $x$ negative. When $x$ is positive (i.e., on the planetary night side), $r_{n}$ must be less than 1 , which means that the hovering radius is less than the Hill radius. When $x$ is negative (on the planetary day side), $r_{n}$ must be greater than 1 , which means that the hovering radius is greater than the Hill radius.

\section{Hovering points in the $x z$-plane}

Now consider when the sail is in the $x z$-plane. Here, $\phi$ and $\psi$ are both 0 while $\alpha$ varies from 0 to $\pi / 2$ as discussed above. In this case, the equations of motion are as in (9)-(11) where

$$
\begin{aligned}
& a_{x}=a_{p} \cos ^{2} \alpha \cos \alpha \\
& a_{y}=0 \\
& a_{z}=a_{p} \cos ^{2} \alpha \sin \alpha
\end{aligned}
$$

We see immediately that $y$ must be 0 from (10). 
Letting $x=r \cos \theta$ and $z=r \sin \theta$, we can solve for angle $\alpha$ and $a_{p}$ in terms of $r$ and $\theta$. Rewriting (9) and (11) they become

$$
\begin{aligned}
\left(\frac{\mu}{r^{3}}-3 \Omega^{2}\right) r \cos \theta & =a_{p} \cos ^{2} \alpha \cos \alpha \\
\left(\frac{\mu}{r^{3}}+\Omega^{2}\right) r \sin \theta & =a_{p} \cos ^{2} \alpha \sin \alpha
\end{aligned}
$$

which gives

$\tan \alpha=\left(\frac{\frac{\mu}{r^{3}}+\Omega^{2}}{\frac{\mu}{r^{3}}-3 \Omega^{2}}\right) \tan \theta$

Multiplying the numerator and denominator by $3 r^{3} / \mu$, we have

$\tan \alpha=\left(\frac{1+\frac{1}{3} r_{n}^{3}}{1-r_{n}^{3}}\right) \tan \theta$

which gives the sail orientation as a function of sail position.

Using the identity $\sec ^{2} \alpha=1+\tan ^{2} \alpha,(9)$ becomes $\frac{\mu}{r^{2}}\left(1-r_{n}^{3}\right) \cos \theta=a_{p}\left(1+\tan ^{2} \alpha\right)^{-3 / 2}$

and, substituting in (25) and solving for $a_{p}$, we have

$$
\begin{aligned}
a_{p}= & \frac{\mu}{r^{2}\left(1-r_{n}^{3}\right)^{2} \cos ^{2} \theta} \times \\
& {\left[\left(1-r_{n}^{3}\right)^{2}+\frac{8}{3} r_{n}^{3} \sin ^{2} \theta\left(1-\frac{1}{3} r_{n}^{3}\right)\right]^{3 / 2} }
\end{aligned}
$$

which gives the necessary sail acceleration as a function of sail position. We can normalize $a_{p}$ completely by dividing by $\mu / r_{H}^{2}$. We then find

$$
\begin{aligned}
\tilde{a}_{p} & =\frac{a_{p}}{\mu / r_{H}^{2}} \\
& =\frac{1}{r_{n}^{2}\left(1-r_{n}^{3}\right)^{2} \cos ^{2} \theta} \times \\
& {\left[\left(1-r_{n}^{3}\right)^{2}+\frac{8}{3} r_{n}^{3} \sin ^{2} \theta\left(1-\frac{1}{3} r_{n}^{3}\right)\right]^{3 / 2} } \\
& \frac{1}{x_{n}^{2}\left(1-r_{n}^{3}\right)^{2}} \times \\
& {\left[\left(1-r_{n}^{3}\right)^{2}+\frac{8}{3} r_{n} z_{n}^{2}\left(1-\frac{1}{3} r_{n}^{3}\right)\right]^{3 / 2} }
\end{aligned}
$$

where $x_{n}=r_{n} \cos \theta$ and $z_{n}=r_{n} \sin \theta$.

When $r_{n} \ll 1$, i.e., the solar sail is close to the asteroid compared to its ideal Hill radius, then

$\tan \alpha \sim \tan \theta\left(1+\frac{4}{3} r_{n}^{3}+\ldots\right)$

and

$a_{p} \sim \frac{\mu}{r^{2} \cos ^{2} \theta}\left(1+\left(4 \sin ^{2} \theta-1\right) r_{n}^{3}+\ldots\right)$

In this case the perturbation due to the solar tidal terms has a small effect on the motion of the spacecraft and is characterized by the value of $r_{11}^{3}$.

\section{Out-of-plane hovering points}

For out-of-plane hovering points, we must take into consideration the most general form of the equations of motion at equilibrium with the equations for SRP and the equation for $\mathbf{r}$ as given above, viz.

$0=-\frac{\mu}{r^{3}} r \cos \theta \cos \psi+3 \Omega^{2} r \cos \theta \cos \psi+a_{x}$
$0=-\frac{\mu}{r^{3}} r \cos \theta \sin \psi+a_{y}$
$0=-\frac{\mu}{r^{3}} r \sin \theta-\Omega^{2} r \sin \theta+a_{z}$

with

$a_{x}=a_{p} \cos ^{2} \phi \cos ^{2} \alpha \cos \phi \cos \alpha$

$a_{y}=a_{p} \cos ^{2} \phi \cos ^{2} \alpha \sin \phi \cos \alpha$

$a_{z}=a_{p} \cos ^{2} \phi \cos ^{2} \alpha \sin \alpha$

Dividing (34) by (33), we find that

$\tan \phi=\frac{1}{1-r_{\imath}^{3}} \tan \psi$

From this equation, we see that angle $\phi$ is related to angle $\psi$ alone, which indicates that as the sail moves out of the $x z$-plane it must also turn so that the sail normal is always pointing approximately radially outward from the asteroid.

Next, we want to solve for angle $\alpha$ and $a_{p}$ as in the previous section, so we again use the identity $1+\tan ^{2} \phi=\sec ^{2} \phi$ to find that

$\sec \phi=\left[\frac{\left(1-r_{n}^{3}\right)^{2}+\tan ^{2} \psi}{\left(1-r_{n}^{3}\right)^{2}}\right]^{1 / 2}$

Dividing (35) by (33) we arrive at the relation

$$
\begin{aligned}
\tan \alpha & =\left(1+\frac{1}{3} r_{n}^{3}\right) \times \\
& {\left[\left(1-r_{n}^{3}\right)^{2}+\tan ^{2} \psi\right]^{-1 / 2} \sec \psi \tan \theta }
\end{aligned}
$$

Solving for $a_{p}$ from (33), we have

$$
\begin{aligned}
& \left(\frac{\mu}{r^{3}}-3 \Omega^{2}\right) r \cos \theta \cos \psi= \\
& \quad a_{p}\left(1+\tan ^{2} \phi\right)^{-3 / 2}\left(1+\tan ^{2} \alpha\right)^{-3 / 2}
\end{aligned}
$$

which becomes

$$
\begin{aligned}
a_{p}= & \frac{\mu}{r^{2}\left(1-r_{n}^{3}\right)^{2} \cos ^{2} \theta \cos ^{2} \psi} W^{3 / 2} \\
W= & \left(1-r_{n}^{3}\right)^{2}+\frac{8}{3} r_{n}^{3} \sin ^{2} \theta\left(1-\frac{1}{3} r_{n}^{3}\right)+ \\
& r_{n}^{3}\left(2-r_{n}^{3}\right) \cos ^{2} \theta \sin ^{2} \psi
\end{aligned}
$$


And, after normalizing completely by dividing by $\mu / r_{H}^{2}$ as before, we have

$$
\begin{aligned}
\tilde{a}_{p}= & \frac{1}{x_{n}^{2}\left(1-r_{n}^{3}\right)^{2}} \tilde{W}^{3 / 2} \\
\tilde{W}= & \left(1-r_{n}^{3}\right)^{2}+\frac{8}{3} r_{n} z_{n}^{2}\left(1-\frac{1}{3} r_{n}^{3}\right)+ \\
& r_{n} y_{n}^{2}\left(2-r_{n}^{3}\right)
\end{aligned}
$$

again giving the sail acceleration and orientation as a function of sail position. In this case, however, we define the normalized position coordinates as $x_{n}=$ $r_{n} \cos \theta \cos \psi, y_{n}=r_{n} \cos \theta \sin \psi$, and $z_{n}=r_{n} \sin \theta$.

Once again, when $r_{n} \ll 1$, we can expand each function about $r_{n}$ and find

$$
\begin{aligned}
\tan \phi \sim & \tan \psi\left(1+r_{n}^{3}+\ldots\right) \\
\tan \alpha \sim & \frac{1}{3} \tan \theta \times \\
& {\left[3+r_{n}^{3}\left(1+3 \cos ^{2} \psi\right)+\ldots\right] } \\
a_{p} \sim & \frac{\mu}{r^{2}} \sec ^{2} \psi \sec ^{2} \theta\left(1+r_{n}^{3} W_{p}+\ldots\right) \\
W_{p}= & -1+3 \sin ^{2} \psi+ \\
& \left(1+3 \cos ^{2} \psi\right) \sin ^{2} \theta
\end{aligned}
$$

Notice that when $\psi=0$ the approximations for $\tan \alpha$ and $a_{p}$ revert back to those found in the $x z$-plane.

Practical consideration of the equilibrium points

The maximum characteristic acceleration possible for the spacecraft upon leaving earth is determined by the mass to area ratio of the sail. In close proximity to the asteroid, the maximum characteristic acceleration varies inversely with the square of the asteroid's distance from the sun. For a given sail acceleration $\tilde{a}_{p},(30)$ or $(45)$ determine the position of the sail needed to hover above the asteroid. This result fully accounts for the effect of the tidal forces acting on the sail, since the general formula gives us results up to $r_{n} \sim 1$.

Focusing on the in-plane case, from (30), for equilibria which lie on the asteroid night side, we see that it takes an infinitely large $\vec{a}_{p}$ to hover around the point where $r_{n}=1$, unless $z_{n}=0$, in which case the necessary $\tilde{a}_{p}$ will drop to 0 . This happens at the equilibrium point along the $+x$-axis which lies in the planet's shadow. It is highly unstable since small errors in the necessary sail acceleration will create large changes in position. We can also see from (30) that we cannot hover outside of the "Hill radius," $r_{n}=1$, as the tidal and SRP forces then cause the sail to escape. We can understand how $\tilde{a}_{p}$ approaches $\infty$ as $r_{n}$ goes to 1 by looking at (25). As $r_{n}$ approaches $1, \tan \alpha$ tends to $\infty$, which implies that $\alpha$ goes to $\pi / 2$ as we approach this radius.

Conversely, on the planetary day side, i.e., $\pi / 2<$ $\theta \leq \pi, r_{n}$ must be greater than 1 in order to achieve equilibrium since $\tan \theta$ is negative in this region. As $r_{n}$ approaches 1 from the left, $\tilde{a}_{p}$ again goes to infinity unless $z_{n}$ is 0 . This occurs at the equilibrium point on the $-x$-axis, indicating a high sensitivity of the hovering point to errors in the vicinity of this point.

The normalized sail acceleration $\tilde{a}_{p}$ is only a function of the sail's characteristic acceleration and the asteroid's gravitational parameter, and is independent of the asteroid's distance from the sun. We see this by expanding the definition for $\tilde{a}_{p}$ :

$\tilde{a}_{p}=\frac{a_{p}}{\left(9 \mu \Omega^{4}\right)^{1 / 3}}$

which, when we combine with (6), gives

$\tilde{a}_{p}=\frac{a_{p o} R_{o}^{2}}{\left(9 \mu \Omega^{4} R^{6}\right)^{1 / 3}}$

For an asteroid in a circular orbit, however, we have $\Omega=\sqrt{\mu_{\text {sun }} / R^{3}}$. Therefore, the relation becomes

$\tilde{a}_{p}=\frac{a_{p o} R_{o}^{2}}{\left(9 \mu \mu_{s u n}^{2}\right)^{1 / 3}}$

or, in terms of the sail lightness number from (8),

$\tilde{a}_{p}=\beta\left(\frac{\mu_{s u n}}{9 \mu}\right)^{1 / 3}$

This form of $\tilde{a}_{p}$ relates the normalized value of $a_{p}$ to the asteroid mass and the sail's characteristic acceleration.

Now, for a given asteroid mass and sail position $\left(x_{n}, z_{n}\right)$, a range of characteristic accelerations can be obtained as shown in Figure 2. These results are comparable to the contours of sail lightness numbers which McInnes ${ }^{2}$ found.

A sail with a given characteristic acceleration (which is transformed into an $\tilde{a}_{p}$ at a given asteroid) can ideally move along lines of constant contour by modifying the orientation of the sail. Thus, the line of constant $\tilde{a}_{p}$ defines the hovering positions that a sail can have at an asteroid. The asteroids which we are using for the purposes of this paper have $\tilde{a}_{p}$ values of approximately 170 for Vesta, 533 for Eros, and 1331 for Ida with corresponding $\beta$ values of 0.1686 , 0.0169 , and 0.0843 , respectively.

In planning multiple-objective missions to asteroids, it would be best to determine the sail acceleration necessary for the desired radial distance from the largest asteroid first. Once the maximum characteristic acceleration is chosen nothing can be done 


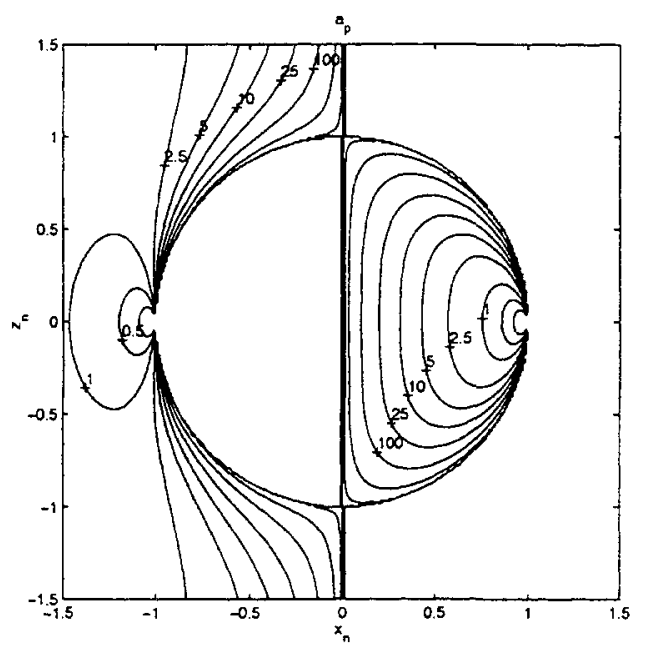

Figure 2: Spacecraft position contours for different values of $\tilde{a}_{p}$. All parameters are nondimensionalized.

to increase it. At the same time, the necessary sail acceleration at the smallest target asteroid must be considered to see what needs to be done for a desired radial distance in terms of either sail attitude, changes in sail area, or both.

Once the target asteroid is determined, we can loosely infer its gravitational acceleration $\mu$ from ground-based observations. In order to find equilibrium points in the $x z$-plane for a particular sail acceleration, we can solve for $r \cos \theta$ from (32) which gives

$r \cos \theta=\sqrt{\frac{\mu}{a_{p}}}+\ldots$

or, dividing by the mean asteroid radius $r_{o}$, we have

$\frac{r}{r_{o}} \cos \theta=\sqrt{\frac{\mu}{a_{p} r_{o}^{2}}}+\ldots$

Equilibrium points can be found when $r / r_{0}>1$ This means that even if $\mu$ is small compared to the sail acceleration, angle $\theta$ can be chosen large enough so that equilibrium points can be found out of the asteroid orbital plane. Similar but more complicated solutions exist for the 3-dimensional case, e.g., (47)(50).

The equilibrium points found here correspond to the stationary solutions located near the earth found by McInnes ${ }^{2}$. McInnes also determined that these hovering points were unstable in the Lyapunov sense but controllable using feedback control to sail attitude alone. Instability and controllability can be established similarly here since McInnes' results satisfied equations which took into account a general form for the potential.

The existence and position of equilibrium points is of interest for station-keeping purposes. These points could be used, for example, in sample return missions where a sail may hover for some period of time while a lander gathers samples and then redocks with the spacecraft. These points also provide positions in which the spacecraft could monitor one area of the asteroid at a constant phase angle for a substantial length of time.

\section{Orbital constraints and dynamics}

We now explore orbital options for the sail about the asteroid. We look for orbits which are stable in the sense that they neither impact the asteroid nor escape. Here we use results from Mignard and Hénon ${ }^{4}$, Richter and Keller ${ }^{5}$, and Scheeres ${ }^{7}$ to identify a family of stable orbits for a solar sail. There are constraints on the orbit geometry and orbit size (as a function of the sail acceleration) for these trajectories to be feasible. These constraints are presented as a function of basic asteroid parameters and heliocentric orbit. When these constraints are met and the orbits are feasible they have a number of features that make them attractive - including better asteroid coverage and nearly trivial station-keeping costs. In this section we will assume $\alpha=\phi=0$ so that the sail is face-on to the sun. For this case, then, $\mathbf{a}=\left[\begin{array}{lll}a_{p} & 0 & 0\end{array}\right]$.

\section{$\underline{\text { Sail constraints for bounded orbits }}$}

A sufficient condition for the orbit semi-major axis of a spacecraft subject to large solar radiation pressure perturbations to be orbitally bound to an asteroid can be derived to be (Hamilton and Burns ${ }^{1}$, Scheeres and Marzari ${ }^{8}$ ):

$a_{s c}<\frac{\tilde{d}}{4} \sqrt{\frac{\mu}{a_{p_{o}}}}$

where $a_{s c}$ is the orbit semi-major axis and $\tilde{d}$ is the asteroid-sun distance in $\mathrm{AU}$. This equation places a restriction on the size of an orbit as a function of the asteroid mass, distance from the sun, and the characteristic acceleration of the sail.

Let us define the normalized semi-major axis $\tilde{a}_{s c}=$ $a_{s c} / r_{0}$ and the maximum sail acceleration for feasible orbital operations:

$a_{p_{0}}^{M}=\frac{\tilde{d}^{2}}{16} \frac{\mu}{r_{o}^{2}}$ 
Then we can define a limiting value of the sail's characteristic acceleration as a function of the maximum sail acceleration $a_{p_{o}}^{M}$ and the desired orbit semimajor axis $\tilde{a}_{s c}$ :

$a_{p_{o}}<\frac{1}{\tilde{a}_{s c}^{2}} a_{p_{o}}^{M}$

where $\tilde{a}_{s c}>1$ in general. Some characteristic values of the parameter $a_{p_{\circ}}^{M}$ are shown for different asteroids in Table 1. This indicates that smaller asteroids will require low values of sail acceleration for orbital options to be feasible about them.

\begin{tabular}{||l|r|r|r|c||}
\hline \multirow{2}{*}{} & \multicolumn{2}{|c|}{$\mathrm{km}$} & \multicolumn{1}{c|}{$\mathrm{km}^{3} / \mathrm{s}^{2}$} & \multicolumn{1}{c|}{$\mathrm{AU}$} \\
\cline { 2 - 5 } & \multicolumn{1}{|c|}{$r_{o}$} & \multicolumn{1}{c|}{$r_{H}$} & \multicolumn{1}{c|}{$\mu$} & $\bar{d}$ \\
\hline & & & & \\
Vesta & 244.27 & 116,680 & 14.2568 & 2.36 \\
Eros & 8.87 & 2,308 & 0.0005 & 1.46 \\
Ida & 15.65 & 9,064 & 0.0038 & 2.86 \\
\hline
\end{tabular}

\begin{tabular}{||l|r|r|r||}
\hline & \multicolumn{3}{|c|}{$\mathrm{mm} / \mathrm{s}^{2}$} \\
\cline { 2 - 4 } & $a_{p_{o}}^{M}$ & $a_{v_{o}}^{m}$ & $\mu / r_{H}^{2}$ \\
\hline & & & \\
Vesta & 83.17 & 0.0494 & 0.0105 \\
Eros & 0.79 & 0.0012 & 0.0009 \\
Ida & 7.83 & 0.0035 & 0.0005 \\
\hline
\end{tabular}

For a sail orbit that is bound, according to the above expression, it has been found ${ }^{4}$ that the semimajor axis of the orbit is constant, on average. The other mean orbit elements are not constant, however, and in general will have large secular variations over time. We confirm these findings in our numerical integrations as well.

\section{Sail orbit dynamics about asteroids}

Assuming we have a solar sail with characteristic acceleration less than the asteroid parameter, it becomes feasible to discuss orbital operations of the sail about the asteroid. The general orbital dynamics of such a bound, highly perturbed orbit are discussed in greater detail in Mignard and Hénon ${ }^{4}$, Richter and Keller ${ }^{5}$, and Scheeres ${ }^{7}$. In Scheeres ${ }^{7}$ a particular class of stable orbits in the highly perturbed SRP problem were identified which are particularly well suited to solar sails. These orbits lie in the plane perpendicular to the asteroid-sun line, nominally have their periapsis aligned $90^{\circ}$ above or below the orbit plane and have their eccentricity chosen according to the equation:

$$
\begin{aligned}
e & =\cos \nu \\
\tan \nu & =\frac{3}{2} a_{p_{o}} d_{o}^{2} \sqrt{\frac{a_{s c}}{P \mu \mu_{s u n}}}
\end{aligned}
$$

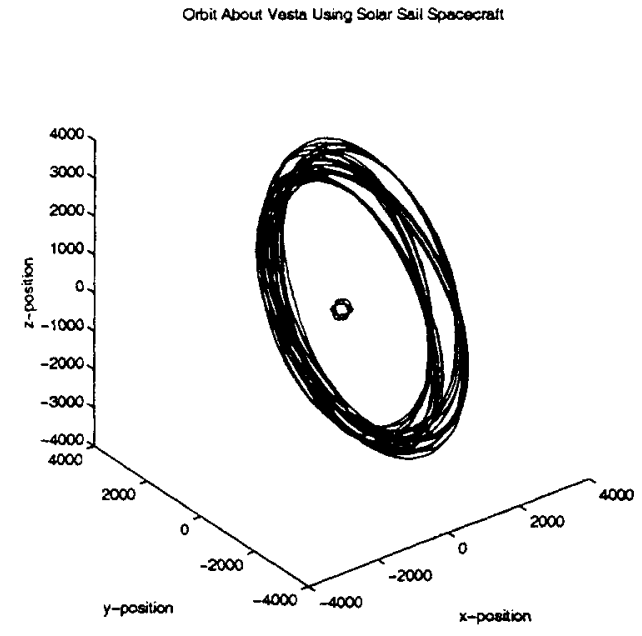

Figure 3: Integrated orbit for a sail about Vesta using orbital elements $a_{s c}=13 r_{o}, e=\omega=\nu_{o}=0$, $i=\Omega_{o}=\pi / 2$; and parameters $r_{o}=244.27 \mathrm{~km}$, $0<t<116$ days and $a_{p_{o}}=1 \mathrm{~mm} / \mathrm{s}^{2}$. Spacecraft position units are in $\mathrm{km}$.

where $d_{o}$ is one astronomical unit in kilometers and $P$ is the orbit parameter of the asteroid, equal to the heliocentric radius for a circular orbit. We can rewrite this result as:

$$
\begin{aligned}
\tan \nu & =\sqrt{\tilde{a}_{s c}} a_{p_{o}} / a_{p_{o}}^{m} \\
a_{p_{o}}^{m} & =\frac{2}{3} \sqrt{\frac{\mu_{s u n}}{d_{o}^{3}}} \sqrt{\frac{\tilde{d} \mu}{r_{o}}} \\
& \sim 1.33 \times 10^{-7} \sqrt{\frac{\tilde{d} \mu}{r_{o}}}
\end{aligned}
$$

where $\tilde{d}$ is the asteroid-sun distance in astronomical units. Some characteristic values of $a_{p_{\circ}}^{m}$ for some select asteroids are shown in Table 1. From these values we note that $\tan \nu \gg 1$ in general, indicating that the stable orbits will have near zero eccentricity.

Shown in Figures 3-7 are a sample of numerically integrated sail trajectories about an asteroid, with the sail placed in a stable orbit as described above. It is important to note that the integration occurs in the frame rotating with the asteroid-sun line. This implies that these orbits are sun-synchronous in that they remain fixed in the sun plane-of-sky as shown in Figure 4. We also note that the trajectory oscillates about a circular orbit in general but appears fixed, as shown in Figure 5.

We can extend the analytical solution of the sail trajectory about the asteroid beyond this simple circular orbit, relying on an analytical solution to the 


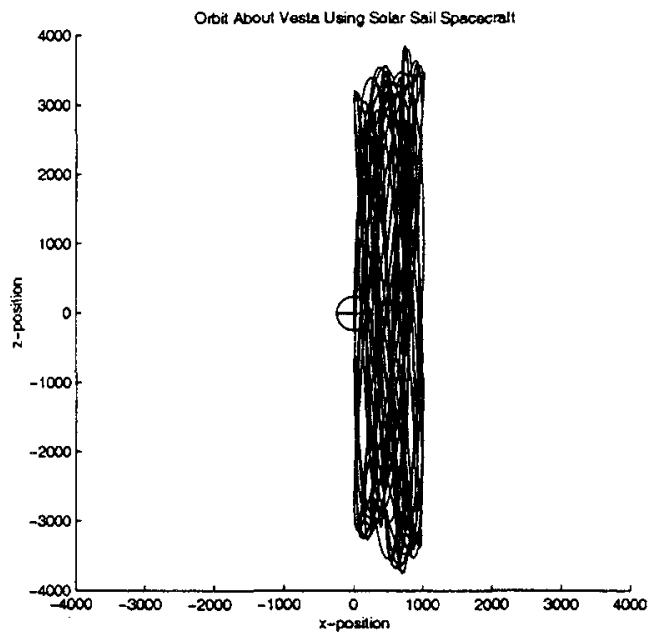

Figure 4: Integrated orbit for a sail about Vesta in the $x z$-plane using orbital elements and parameters of figure 3 .

averaged solar radiation pressure problem formulated by Richter and Keller. ${ }^{5}$. In this paper they find the averaged angular momentum and periapsis vector of the orbit as a function of asteroid true anomaly with respect to the sun. Taking this general solution, we specialize it to our particular case by assuming that the perturbation angle $\nu \rightarrow \pi / 2$. We then find the angular momentum vector and periapsis vector to be:

$$
\begin{aligned}
\mathbf{H} & =\sqrt{\mu a}\left[\begin{array}{c}
C_{1} \\
C_{2} \sin (H)+C_{3} \cos (H) \\
C_{5} \cos (H)-C_{6} \sin (H)
\end{array}\right] \\
\mathbf{e} & =-\left[\begin{array}{c}
C_{4} \\
C_{5} \sin (H)+C_{6} \cos (H) \\
C_{2} \cos (H)-C_{3} \sin (H)
\end{array}\right]
\end{aligned}
$$

where $H$ is an angle proportional to the asteroid true anomaly about the sun. The constants $C_{i}$ can be related to the initial osculating orbital elements of the sail orbit as:

$$
\begin{aligned}
& C_{1}=\sqrt{1-e^{2}} \sin i \sin \Omega \\
& C_{2}=-e \sin i \sin \omega \\
& C_{3}=-\sqrt{1-e^{2}} \sin i \cos \Omega \\
& C_{4}=-e[\cos \Omega \cos \omega-\cos i \sin \Omega \sin \omega] \\
& C_{5}=\sqrt{1-e^{2}} \cos i \\
& C_{6}=-e[\sin \Omega \cos \omega+\cos i \cos \Omega \sin \omega]
\end{aligned}
$$

From this solution it is possible to evaluate the evolution of the mean orbit elements as a function of $H \sim n t / \cos \nu$, where $n$ is the asteroid's mean motion

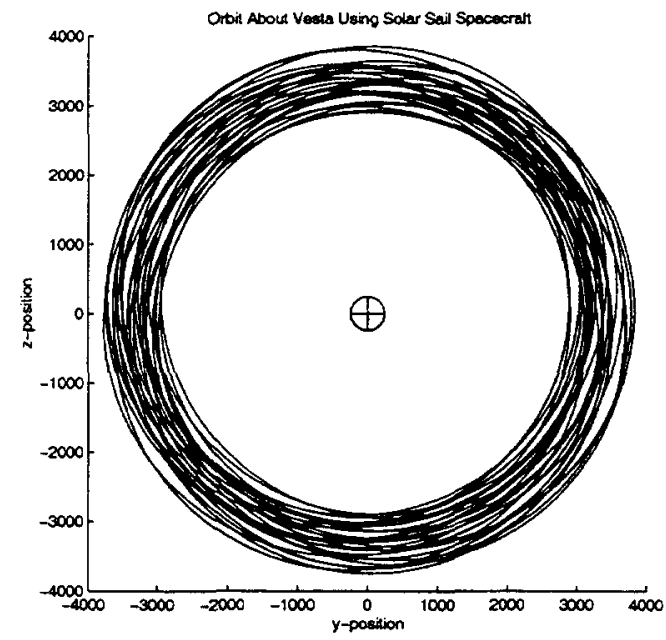

Figure 5: Integrated orbit for a sail about Vesta in the $y z$-plane using orbital elements and parameters of figure 3 .

about the sun, $t$ is time, and $\nu$ is the perturbation angle again. We note that for $\nu=\pi / 2-\epsilon$, which is our case, the angle $H$ will increase very rapidly with time, indicating that the mean solutions will oscillate rapidly. This is not necessarily desirable, as can be seen if we take as initial conditions $i=0, e=0$, giving $C_{1}=C_{2}=C_{3}=C_{4}=C_{6}=0$, and $C_{5}=1$. The solution then becomes:

$$
\begin{aligned}
e & =|\sin (H)| \\
\omega & = \pm \pi / 2 \\
i & =0
\end{aligned}
$$

meaning that the orbit will remain in the ecliptic plane and the eccentricity will repeatedly pass through a value of unity, eventually causing the sail to impact on the asteroid surface.

If instead we take initial conditions $i=\pi / 2, e=0$, $\Omega=\Omega_{o}$, we find the solution:

$$
\begin{aligned}
i & =\pi / 2 \\
\omega & = \pm \pi / 2 \\
e & =\left|\cos \Omega_{o}\right||\sin (H)| \\
\tan \Omega & =\frac{\tan \Omega_{o}}{\cos (H)}
\end{aligned}
$$

Now the orbit plane remains normal to the asteroid ecliptic, the longitude of the ascending node varies between $\Omega_{o} \leq \Omega \leq \pi-\Omega_{o}$, and the eccentricity varies between $0 \leq e \leq\left|\cos \Omega_{0}\right|$. This is a potentially useful generalization of the previously discussed stable circular orbit that allows the orbit 


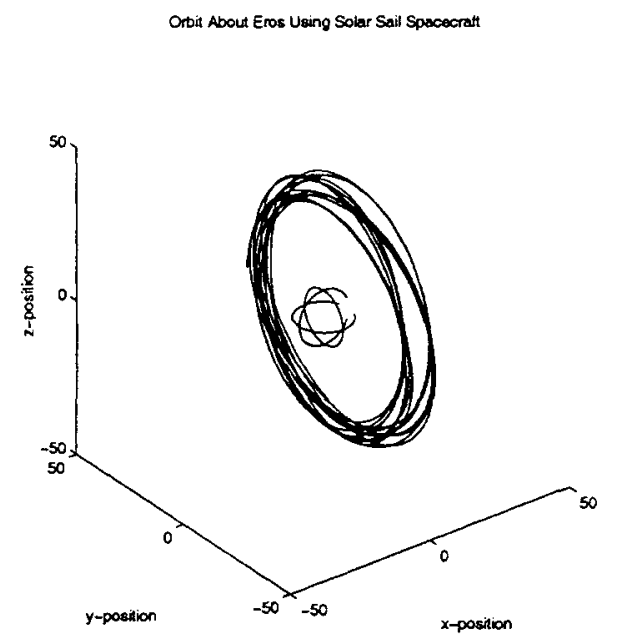

Figure 6: Integrated orbit for a sail about Eros with $a_{s c}=4 r_{o} ; e, i, \omega, \Omega_{o}$ and $\nu_{o}$ the same as in Fig. 3; $r_{o}=8.87 \mathrm{~km} ; 0<t<12$ days; and $a_{p_{o}}=.1 \mathrm{~mm} / \mathrm{s}^{2}$.

plane to move out of the sun plane-of-sky, while bounding the maximum value of eccentricity. In Figure 8 we show another plot of a numerically integrated orbit which shows this characteristic solution. Note that, even though the oscillations in mean elements occur rapidly, it is not valid to average over them, as the amplitude of the oscillations are large. In Figure 9 we show how eccentricity varies with $\Omega$ and is bounded.

This family of solutions provide a viable family of stable, sun synchronous sail orbits about asteroids in general that require no active control to maintain and which allows the sail to view the asteroid over a larger range of phase angles and for long periods of time. Since these orbits are stable, we do not need to apply any closed loop control to stabilize the sail, which implies that the orbital operations will be safer and require less operational effort.

\section{Conclusion}

We have shown that both hovering and orbital options are available with a solar sail spacecraft operating in close proximity to a spherical asteroid in a circular orbit. There are a continuum of hovering positions available near the asteroid which are unstable. They can be stabilized, however, using a feedback control loop to sail attitude alone. These hovering points depend on sail attitude, asteroid mass, and the characteristic acceleration of the sail. For a given distance from the asteroid and characteristic acceleration, the sail attitude can be determined to maintain that position for extended periods of time.

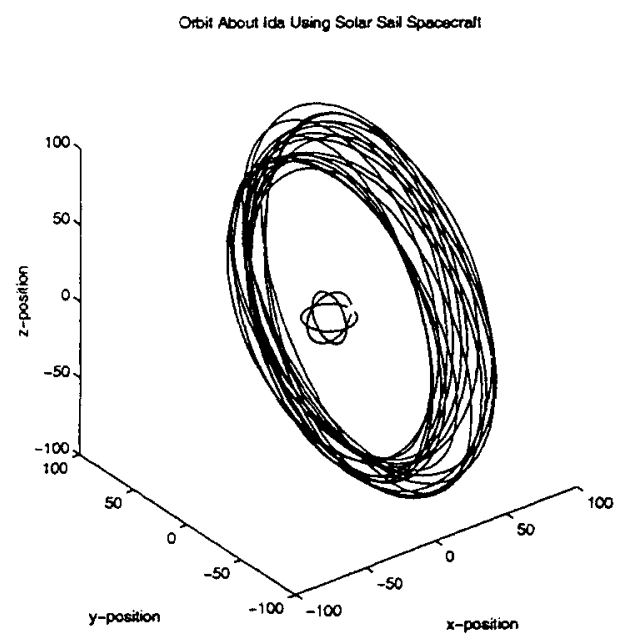

Figure 7: Integrated orbit for a sail about Ida with $a_{s c}=6 r_{o} ; e, i, \omega, \Omega_{o}$ and $\nu_{o}$ the same as in Fig. $3 ; r_{\circ}=15.65 \mathrm{~km} ; 0<t<24$ days; and $a_{p_{o}}=.5$ $\mathrm{mm} / \mathrm{s}^{2}$.

It has also been shown that several orbital options are available which offer good coverage of the asteroid and which neither impact the surface nor escape. These orbits are stable and sun synchronous. A sail could maintain orbit for extended periods of time in such an orbit. Some asteroids (such as Eros) require very small characteristic accelerations for orbiting to be feasible. In such cases as these where it may be necessary to reduce the sail acceleration, we can make adjustments with trims in the sail area. With the possible exception of spin-stabilized sails, the deployment mechanism could be reversed so that the sail could be partially refurled. In this way we could decrease the sail area and thereby decrease $a_{p}$, making orbital operations about smaller asteroids possible.

Solar sails offer the advantage that long-duration missions can be planned, visiting several solar system objects, without the spacecraft mass and complexity involved with conventional propulsion and fuel supply. For example, after one asteroid has been investigated, the spacecraft is capable of escaping from orbit, in principle under sail propulsion alone, and traveling to another or returning to earth. This capability offers the potential for low-cost and flexible solar system exploration.

\section{Acknowledgements}

Research at the University of California, San Diego, was supported by the National Science Foun- 


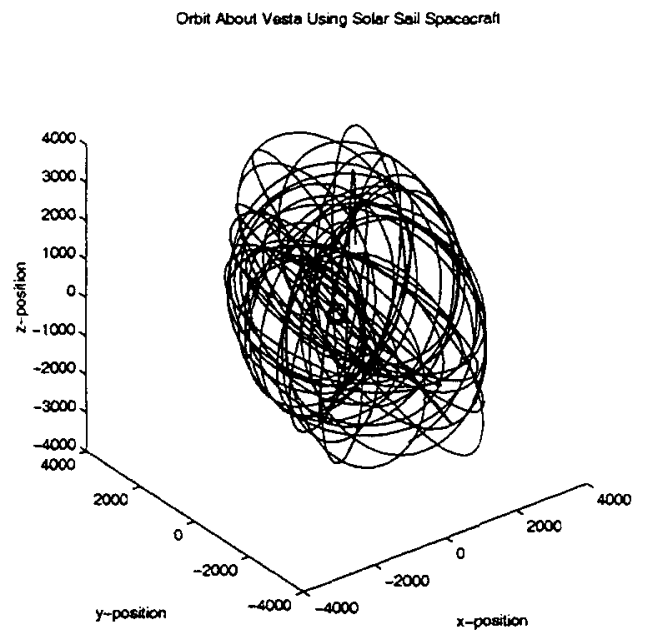

Figure 8: Integrated orbit for a sail about Vesta. $a_{s c}, e, i, \omega, \nu_{o}$ and $a_{p_{o}}$ same as in Fig. $3 ; \Omega_{o}=45^{\circ}$; $0<t<116$ days

dation NSF-OPP 9806941 . Research at The University of Michigan was supported by the TMOD Technology Program by a grant from the Jet Propulsion Laboratory, California Institute of Technology which is under contract with the National Aeronautics and Space Administration.

\section{Bibliography}

[1] Hamilton, D.P. and Burns, J.A. 1992. "Orbital Stability Zones about Asteroids II. The destabilizing effects of eccentricities and or solar radiation," Icarus 96, 43-64.

[2] McInnes, C.R., A.J.C. McDonald, J.F.L. Simmons, E.W. MacDonald. 1994. "Solar Sail Parking in Restricted Three-Body Systems," Journal of Guidance, Control, and Dynamics Vol. 17, No. 2, 399-406.

[3] McInnes, C.R. 1999. Solar Sailing: technology, dynamics and mission applications. Chichester, UK

[4] Mignard, F. and Hènon, M. 1984. "About an Unsuspected Integrable Problem," Celestial $\mathrm{Me}$ chanics 33, $239-250$.

[5] Richter, K. and Keller, H.U. 1995. "On the stability of dust particle orbits around cometary nuclei," Icarus 114, 355-371.

[6] Scheeres, D.J. 1998. "The Restricted Hill Four-Body Problem with Applications to the EarthMoon-Sun System," Celestial Mechanics and Dynamical Astronomy 70, 75-98.

[7] Scheeres, D.J. 1999. "Satellite Dynamics about Small Bodies: Averaged Solar Radiation Pressure

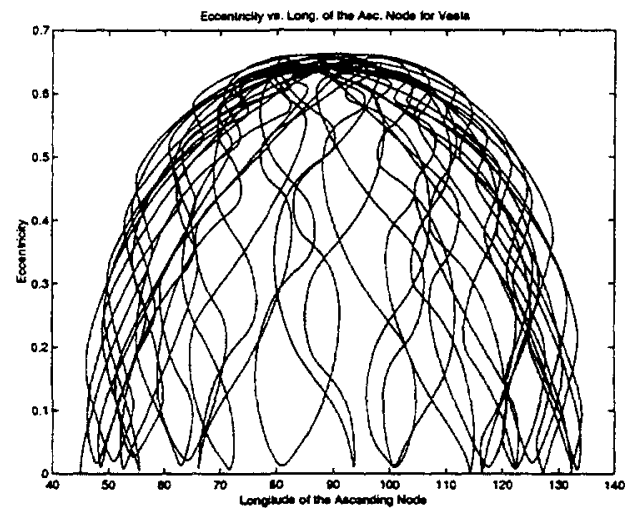

Figure 9: Eccentricity vs. Longitude of the Ascending Node for Orbit about Vesta.

Effects," Journal of the Astronautical Sciences 47, $25-46$.

[8] Scheeres, D.J., F. Marzari. 2000. "Spacecraft dynamics far from a comet." Submitted to the Journal of Astronautical Sciences. 